Role of effective atomic masses in memory function-based models for liquids: A simulation study of liquid water

Vania Calandrini, Godehard Sutmann, Antonio Deriu, and Gerald R. Kneller

Citation: The Journal of Chemical Physics 125, 236102 (2006); doi: 10.1063/1.2403877

View online: https://doi.org/10.1063/1.2403877

View Table of Contents: http://aip.scitation.org/toc/jcp/125/23

Published by the American Institute of Physics 


\title{
Role of effective atomic masses in memory function-based models for liquids: A simulation study of liquid water
}

\author{
Vania Calandrini \\ Laboratoire Léon Brilloin, CEA Saclay, 91191 Gif-sur-Yvette, France \\ Godehard Sutmann \\ Central Institute for Applied Mathematics (ZAM), Research Centre Jülich, D-5242 Jülich, Germany and \\ John von Neumann Institute for Computing (NIC), Research Centre Jülich, D-5242 Jülich, Germany \\ Antonio Deriu \\ Physics Department, Universitá di Parma, Via G. P. Usberti 7/A, I-43100 Parma, Italy \\ Gerald R. Kneller ${ }^{\mathrm{a}), \mathrm{b})}$ \\ Laboratoire Léon Brilloin, CEA Saclay, 91191 Gif-sur-Yvette, France and Centre de Biophysique \\ Moléculaire, CNRS, Rue Charles Sadron, 45071 Orléans, France
}

(Received 26 May 2006; accepted 9 November 2006; published online 19 December 2006)

[DOI: $10.1063 / 1.2403877]$

The theoretical description of the dynamics of liquids by the theory of Mori and Zwanzig is in practice mostly applied to simple liquids, where the molecules have no internal structure. ${ }^{1}$ Recently a second-order Mori-Zwanzig model for the incoherent intermediate scattering function has been employed in order to interpret quasielastic neutron scattering (QENS) experiments on water and aqueous solutions of nonpolar molecules. ${ }^{2-4}$ In this framework the time evolution of the single particle correlation function $F(q, t)=\langle\exp [i q(R(t)$ $-R(0))]\rangle$ is described by

$$
\begin{aligned}
& \dot{F}(q, t)=-\int_{0}^{t} d \tau M^{(1)}(q, t-\tau) F(q, \tau), \\
& \dot{M}^{(1)}(q, t)=-\int_{0}^{t} d \tau M^{(2)}(q, t-\tau) M^{(1)}(q, \tau),
\end{aligned}
$$

where $M^{(1)}(q, t)$ and $M^{(2)}(q, t)$ are, respectively, the first and second order memory functions. Their initial values are determined by the short time behavior of $F(q, t)$ ("sum rules"), $M^{(1)}(q, 0)=\left\langle v^{2}\right\rangle q^{2}$, and $M^{(2)}(q, 0)=2\left\langle v^{2}\right\rangle q^{2}+\Omega^{2}$. Here $\Omega^{2}$ $=\left\langle\dot{v}^{2}\right\rangle /\left\langle v^{2}\right\rangle$ is the square of the so-called Einstein frequency. The pivot of the model, which as been used for the interpretation of the QENS data in Ref. 2-4 is a second order memory function in form of a weighted sum of two exponentials, describing, respectively, fast collision-induced relaxation of the single-particle dynamics and slower structural relaxation of the surrounding liquid,

$$
M^{(2)}(q, t)=M^{(2)}(q, 0)\left\{\alpha \exp \left(-t / \tau_{1}\right)+(1-\alpha) \exp \left(-t / \tau_{2}\right)\right\} .
$$

Here $\tau_{1}$ and $\tau_{2}$ are the fast and the slow relaxation times, respectively, and $\alpha$ is a positive number with $0 \leqslant \alpha \leqslant 1$. The dependence on $q$ of these parameters is here omitted. Since QENS is sensitive to slow diffusive long range motions of the water molecules, the latter were treated as point masses, using the predominantly scattering hydrogen atoms as probes for the center-of-mass motion.
In this note we use molecular dynamics (MD) simulations and numerical calculations of memory functions to verify and to extend the validity of the analytical model described above. We show, in particular, that the model can also be used for a more detailed description of the molecular dynamics, where molecules are considered as rigid bodies. In this way both diffusive motions and fast intermolecular vibrations and librations can be accounted for. The rigid-body model corresponds, in particular, to what thermal neutrons "see" in scattering experiments. ${ }^{5}$ The MD simulations have been performed in the same picture, using the simple point charge/extended (SPC/E) potential ${ }^{6}$ in which a rigid body description of the simulated water molecules is employed. The simulated system consisted of 256 water molecules in a cubic box. All simulations have been performed with the simulation program DL_POLY (Version 2), ${ }^{7}$ using Ewald summation for long-range electrostatic interactions and ambient temperature and pressure conditions. To estimate $F(q, t)$, its memory function and its Fourier spectrum from MD trajectories we use autoregressive modeling for the time series of the underlying dynamical variables. ${ }^{8}$ The numerical method for the calculation of higher order memory functions will be described in a forthcoming paper.

Within the rigid-body picture, the mean square velocity of the hydrogen atoms can be expressed by

$$
\left\langle v^{2}\right\rangle=k_{B} T / m_{\text {eff }}(H),
$$

where $m_{\text {eff }}$ is an effective mass depending on the the geometry of the molecule under consideration and on the masses of its atoms. ${ }^{9}$ We note here that the effective mass can be identified with the Sachs-Teller mass of the scattering atom, which describes the inertia of atoms in collisions between rigid molecules and thermal neutrons. ${ }^{5}$ For the SPC/E water model one finds that $m_{\mathrm{eff}}\left(H_{1}\right)=m_{\mathrm{eff}}\left(H_{2}\right)=1.896$, using atomic mass units. Formally, the square of the Einstein frequency is given by 


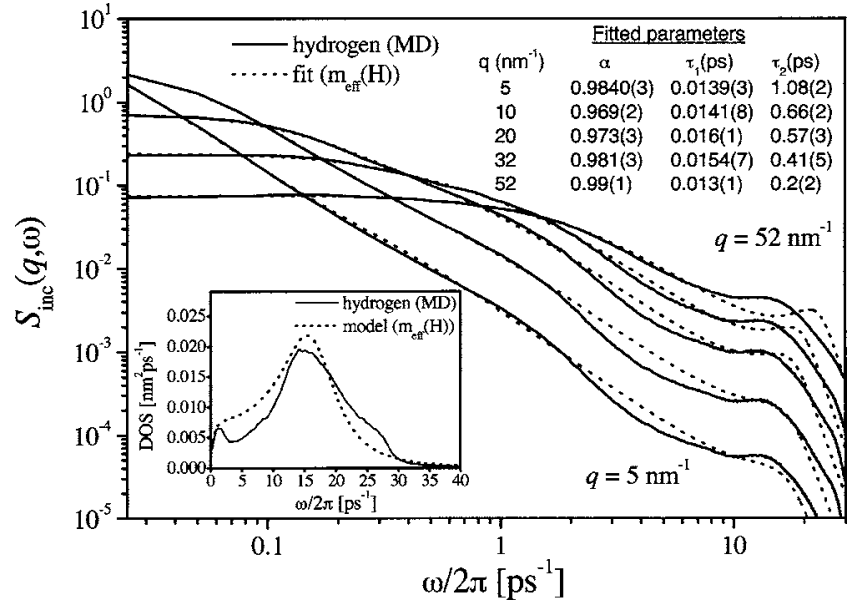

FIG. 1. Log-log plot of $S(q, \omega)$ from MD simulation (solid lines) and the corresponding fits of the analytical model (dotted lines). The inset shows the DOS of the hydrogen atom in water obtained from MD simulation (solid line) and from the analytical model (dotted line). More explanations are given in the text.

$$
\Omega_{H}^{2} \equiv \frac{\left\langle\left(F_{H}+Z_{H}\right)^{2}\right\rangle}{m_{H}^{2}} \frac{m_{\mathrm{eff}}(H)}{k_{B} T},
$$

where $m_{H}=1.008$ is the mass of a free hydrogen atom in atomic mass units and $F_{H}$ and $Z_{H}$ are, respectively, the external force and the constraint force. The latter is due to the geometrical constraints and it should be noted that it contains contributions from inertial forces involving themselves the atomic masses. In the present study we obtained $\Omega_{H}^{2}$ $=11881 \mathrm{ps}^{-2}$ by direct calculation from the MD trajectories, using $\Omega^{2}=M_{v}^{(1)}(0)$, where $M_{v}^{(1)}(t)$ is the first order memory function of the velocity autocorrelation function.

Fixing the parameters $\left\langle v_{H}^{2}\right\rangle$ and $\Omega_{H}^{2}$, we fitted the dynamic structure factor of the model to the simulated incoherent dynamic structure factor, $S(q, \omega)=(2 \pi)^{-1} \int_{-\infty}^{+\infty} d t \exp ($ $-i \omega t) F(q, t)$. The results are shown in Fig. 1, together with the fitted values for $\alpha, \tau_{1}$, and $\tau_{2}$. Using the fit parameters corresponding to $q=5 \mathrm{~nm}^{-1}$ we computed the model density of states (DOS) of the hydrogen atoms, which is given by $g(\omega)=\lim _{q \rightarrow 0}\left(\omega^{2} / q^{2}\right) S(q, \omega)$. Since the limit $q \rightarrow 0$ cannot be performed numerically, we used the smallest possible value $q=5 \mathrm{~nm}^{-1}$ on account of the box size and statistical accuracy. In this approximation we find $D \approx 3.1 \times 10^{-5} \mathrm{~cm}^{2} / \mathrm{s}$ from the simulation, as compared to $D \approx 3.4 \times 10^{-5} \mathrm{~cm}^{2} / \mathrm{s}$ for the model fit. We note that $D \approx 2.55 \times 10^{-5} \mathrm{~cm}^{2} / \mathrm{s}$ is obtained from the slope of the mean square displacement, which is close to the experimental value of $D \approx 2.23 \times 10^{-5} \mathrm{~cm}^{2} / \mathrm{s}$. The above findings show that treating the water molecules as

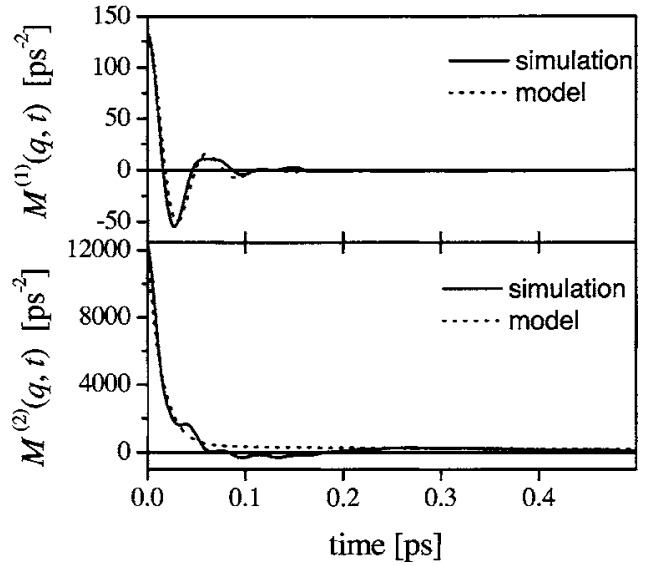

FIG. 2. First order and second order memory function of $F(q, t)$ for $q$ $=10 \mathrm{~nm}^{-1}$, as obtained from MD simulation (solid lines) and from the analytical model (dotted lines), using the parameters given in Fig. 1.

rigid bodies and the predominantly scattering hydrogen atoms as mass points carrying the Sachs-Teller mass, allows us to correctly reproduce not only the quasielastic, diffusive regime, but also the inelastic regime corresponding to intermolecular vibrations and fast librations.

To further test the validity of the model, we computed the corresponding first and second order memory functions. Figure 2 shows as an example the comparison between the memory functions obtained from the model and those computed from MD simulation at $q=10 \mathrm{~nm}^{-1}$. The model memory functions have been calculated using the parameters given in Fig. 1. Figure 2 shows that the first order memory function (top) is remarkably well reproduced by the model, and that the second order memory function (bottom) shows the correct limiting behavior.

${ }^{a)}$ Author to whom correspondence should be addressed. Electronic mail: kneller@cnrs_orleans.fr

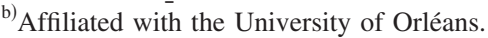

${ }^{1}$ J. P. Boon and S. Yip, Molecular Hydrodynamics (McGraw-Hill, New York, 1980)

${ }^{2}$ V. Calandrini, A. Deriu, G. Onori, R. E. Lechner, and J. Pieper, J. Chem. Phys. 120, 4759 (2004).

${ }^{3}$ D. Di Cola, A. Deriu, and M. Sampoli, Physica B 226, 46 (1996).

${ }^{4}$ V. Calandrini, A. Deriu, G. Onori, A. Paciaroni, and M. T. F. Telling, J. Phys.: Condens. Matter 18, 23 (2006).

${ }^{5}$ R. G. Sachs and E. Teller, Phys. Rev. 60, 18 (1941).

${ }^{6}$ H. J. C. Berendsen, J. R. Grigera, and T. P. Straatsma, J. Phys. Chem. 91, 6269 (1987).

${ }^{7}$ W. Smith, C. W. Yong, and P. M. Rodger, Mol. Simul. 28, 385 (2002).

${ }^{8}$ G. R. Kneller and K. Hinsen, J. Chem. Phys. 115, 11097 (2001).

${ }^{9}$ G. R. Kneller, J. Chem. Phys. 125, 14107 (2006).

${ }^{10}$ K. T. Gillen, D. C. Douglas, and M. J. R. Hoch, J. Chem. Phys. 57, 5117 (1972). 